\title{
Die Netzwerkanalyse und der Umgang mit ihren Forschungsergebnissen. Eine kritische Replik zum Beitrag von Nico Sonntag
}

\author{
Walter Otto Ötsch $\cdot$ Katrin Hirte $\cdot$ Stephan Pühringer
}

Angenommen: 13. Januar 2022 / Online publiziert: 27. Januar 2022

(C) Der/die Autor(en) 2022

Zusammenfassung In der Untersuchung von sozialen Netzwerken werden Beziehungen zwischen sozialen Akteuren (z.B. Personen, Institutionen, Organisationen) adressiert und nach dem Charakter dieser Beziehungen gefragt. Dieser Beziehungscharakter interessiert vor allem dann, wenn die Akteure in diesen Netzwerken gemeinsame inhaltliche Ausrichtungen entwickeln, die performativ in die Gesellschaft hineinwirken. Da in solchen Netzwerkanalysen die relevanten Akteure benannt und deren Denkausrichtungen eruiert werden, bergen sie einerseits ein aufklärerisches Potenzial. Andererseits sind sowohl die Forschenden als auch die Scientific Community beim Umgang mit diesen Forschungsergebnissen angehalten, die Netzwerkbeziehungen und die inhaltlichen Ausrichtungen der Akteure*innen als mehr oder weniger manifeste und prozessierende aufzufassen, anstatt diese bewusst oder unbewusst zu reifizieren. In der vorliegenden Replik setzen wir uns kritisch mit dem in dieser Zeitschrift veröffentlichten Vorwurf Nico Sonntags auseinander, wir wären bei unserer Analyse von ordoliberalen Netzwerken in Deutschland oberflächlich und intransparent vorgegangen, suggerierten die Realität eines kollektiv agierenden marktfundamentalen Netzwerkes und hätten damit womöglich sogar einem Verschwörungsdenken Vorschub geleistet.

\footnotetext{
Walter Otto Ötsch $(\bowtie)$

Institut für Ökonomie, Cusanus Hochschule für Gesellschaftsgestaltung,

Kornpfortstraße 15, 56068 Koblenz, Deutschland

E-Mail: walter.oetsch@cusanus-hochschule.de

Katrin Hirte · Stephan Pühringer

Institut für die Gesamtanalyse der Wirtschaft, Johannes Kepler Universität Linz,

Altenbergerstraße 69, 4040 Linz, Österreich

Katrin Hirte

E-Mail: katrin.hirte@jku.at

Stephan Pühringer

E-Mail: stephan.puehringer@jku.at
} 
Schlüsselwörter Analyse sozialer Netzwerke - Social Studies of Economics · Ordoliberalismus · Ökonomische Politikberatung · Deutsche Ökonom*innen

\title{
Network analysis and the handling of its research results. A critical reply to Nico Sonntag
}

\begin{abstract}
The main aim of social network research is to study relationships between social actors (e.g. individuals, institutions, organizations) as well as the specific character these relationships can take on. The latter is of particular interest when the actors in these networks align to exert a performative impact on society. Thus, by highlighting distinct actors and their connections, social network analyses provide insights into the social structure of networks. However, when dealing with these research results, both researchers and the scientific community need to understand the network relations and intellectual orientations of the actors as more or less manifest and processive, instead of consciously or unconsciously reifying them. In this reply, we critically address Nico Sonntag's allegations, published in this journal, that our analysis of ordoliberal networks in Germany was superficial and non-transparent, that we suggested the reality of a collectively-acting market-fundamental network, and that our results may even have abetted conspiracy thinking.
\end{abstract}

Keywords Social network analysis - Social studies of economics · Ordoliberalism $\cdot$ Economic policy advice $\cdot$ German economists

\section{L'étude des réseaux et l'interprétation des résultats de ses recherches. Une réponse critique à l'article de Nico Sonntag}

Résumé L'étude des réseaux sociaux porte sur les relations entre acteurs sociaux ( $p$. ex. personnes, institutions, organisations) et s'interroge sur la nature de ces relations. La nature de ces relations est particulièrement intéressante quand les acteurs développent au sein de ces réseaux des orientations idéologiques ayant un impact performatif sur la société. En désignant les acteurs pertinents et en étudiant leurs orientations idéologiques, de telles analyses font œuvre de dévoilement. Quand ils interprètent les résultats de ces recherches, les chercheurs et la communauté scientifique sont cependant tenus de concevoir les relations au sein de ces réseaux ainsi que les orientations idéologiques des acteurs comme étant plus ou moins manifestes et processuelles au lieu de les réifier consciemment ou inconsciemment. La présente contribution répond de manière critique aux reproches formulés par Nico Sonntag dans cette revue selon lesquels notre analyse des réseaux ordolibéraux en Allemagne serait superficielle et manquerait de transparence, que nous suggérerions l'existence d'un réseau de partisans radicaux du marché agissant collectivement, voire même que nous aurions de la sorte encouragé la pensée conspirationniste.

Mots-clés Analyse des réseaux sociaux - Social studies of economics . Ordolibéralisme $\cdot$ Conseil en politique économique $\cdot$ Économistes allemand.e.s 


\section{Einleitung}

Mit den nachstehenden Ausführungen beziehen wir uns auf Nico Sonntags kritischen Beitrag „Anmerkungen zum Gebrauch der Netzwerkanalyse in der Neoliberalismusforschung“, der in Heft 1-2/2019 des „Berliner Journals für Soziologie“ veröffentlicht wurde (Sonntag 2019). Darin wird unser Buch Netzwerke des Marktes. Ordoliberalismus als Politische Ökonomie (Ötsch et al. 2018) als ein Beispiel dafür herangezogen, wie eine Netzwerkforschung eher nicht aussehen sollte. Der zentrale Vorwurf lautet, dass wir die Methoden der Netzwerkanalyse ,unbedarft und oberflächlich“ verwendet hätten (Sonntag 2019, S. 154) und die Existenz wirkmächtiger marktfundamentaler Netzwerke unterstellen, ohne deren effektiven Einfluss wirklich belegen zu können. Der Mehrwert unserer Netzwerkanalyse bleibe daher unklar (ebd., S. 164). Die Schwere dieses Vorwurfs verlangt eine Stellungnahme.

Bei Netzwerkanalysen stehen soziale Beziehungen und ihre Ausprägungen im Zentrum des Erkenntnisinteresses. Dabei wird bewusst eine relationale Perspektive eingenommen (Emirbayer 1997). Anstatt von Gruppen von Akteur*innen als unabhängige Einheiten mit gemeinsamen Merkmalsausprägungen auszugehen, bilden die Eingebundenheit der Akteur*innen in soziale Beziehungsstrukturen den Ausgangspunkt der Analyse (Haas und Malang 2010, S. 89). Die Ausbildung des Gemeinsamen in den Beziehungen der Akteur*innen wird somit selbst zum Untersuchungsgegenstand, anstatt es - wie in positionalen Ansätzen typisch (Saunders 2007, S. 227) - einfach vorauszusetzen. Neben der relationalen wird eine prozessuale Perspektive eingenommen, denn zwischen den Positionen in einem Netzwerk und der Art von Beziehungen besteht ein dynamisches Wechselverhältnis: Durch die Art der Beziehungen werden die Positionen erst konstituiert, und zwar in einem Aushandlungsprozess, bei dem die konkreten Beziehungen und Positionen weiter ausgestaltet werden (Stegbauer 2010, S. 136).

Diese Ausgestaltung von Beziehungen und die Ergebnisse von Aushandlungsprozessen geraten vor allem dann verstärkt in den analytischen Fokus, wenn damit gesellschaftlich relevante Entwicklungstendenzen einhergehen, d.h. wenn Netzwerken ein performativer Einfluss auf bestimmte Bereiche der Gesellschaft und insbesondere auf die Politik zugeschrieben wird. Bei der wissenschaftlichen Identifizierung solcher (vermeintlich) einflussreicher Netzwerke und beim Umgang mit den Forschungsergebnissen zu solchen Netzwerken stellen sich besondere Ansprüche an die Forschenden und an die Scientific Community, aber auch an die Öffentlichkeit. Einerseits sind die sozialen Beziehungen der Netzwerkakteur*innen und die Zuschreibungen von inhaltlichen Ausrichtungen solcher Netzwerke als z.B. ,neoliberal“, „ordoliberal“" oder „keynesianisch-gewerkschaftsnah“ seitens der Forschenden empirisch zu eruieren und zu belegen. Andererseits fordern solche Forschungsergebnisse auch das Verständnis solcher Netzwerke bei den Mitgliedern der Scientific Community und der breiteren Öffentlichkeit heraus, die diese Ergebnisse aufnehmen, diskutieren, kritisieren oder affirmieren. Hier können bestimmte Vordisponierungen seitens der Mitglieder der Scientific Community oder der Vertreter der Öffentlichkeit bestehen, die deren vorurteilslosen Blick auf die wissenschaftlichen Ergebnisse einer Netzwerkanalyse und deren begrenzten Geltungsanspruch trüben können. 
Auch in der Kritik von Sonntag an unserer Netzwerkanalyse scheint die eigene Präferenz für quantitativ-formale Netzwerkanalysen insbesondere von direkten sozialen Interaktionen zwischen Personen eine gewichtige Rolle gespielt zu haben. Daher gerät Sonntag, wie wir in unserer Replik zeigen wollen, hinsichtlich unseres netzwerkanalytischen Vorgehens auf die falsche Fährte, weshalb wir, nach einem kurzen Überblick über das Anliegen und den Inhalt unseres Buches (Abschnitt 2), unsere Methode, die zu den von ihm kritisierten Netzwerkabbildungen geführt hat, noch einmal explizit erläutern (Abschnitt 3). Obwohl Sonntag - zu Recht - vor den Gefahren der Reifikation von Kanten und Knoten warnt, neigt er in seiner Kritik an unseren Netzwerkvisualisierungen selbst dazu, solche Abbildungen zu reifizieren und ihnen eine großes Verführungspotenzial zuzuschreiben. Die Replik endet mit einer Zurückweisung der von Sonntag selbst ,lediglich insinuierten Schlussfolgerung[]" (so ein Vorwurf von ihm an unsere Adresse; Sonntag 2019, S. 162), wir würden die Erforschung von Netzwerkbeziehungen im Grunde auf die Aufdeckung von kollektiven Strategien der Allianzbildung verkürzen und durch eine mit dem ,wissenschaftliche[n] Nimbus von Netzwerkmethoden“ versehene Analyse und bildliche Darstellung von Elitenverflechtungen womöglich das ,,verschwörungstheoretische[] Denken“ fördern (ebd., S. 168).

\section{Zur Studie Netzwerke des Marktes}

Die Kritik von Sonntag am Gebrauch der Netzwerkanalyse in der Neoliberalismusforschung zielt vor allem auf unsere Publikation Netzwerke des Marktes (Ötsch et al. 2018). Das Buch ist das Ergebnis zweier Forschungsprojekte' ${ }^{1}$. In ihm wird das ökonomische, politische und gesellschaftliche Wirkungspotenzial von Netzwerken aus Akteur*innen mit verschiedenen wirtschaftspolitischen Grundvorstellungen in der Bundesrepublik Deutschland analysiert. Anlass und Ausgangspunkt der Untersuchung war das konstitutive Spannungsverhältnis zwischen Wissenschaft und Politik in der Ökonomik. Einerseits folgt sie der Selbstbeschreibung, Ökonom*innen ${ }^{2}$ betrieben eine wertfreie positive Wissenschaft, die wirtschaftliche Abläufe modelliere und anschließend die Modelle empirisch überprüfe. Andererseits bestehen zahlreiche wirtschaftspolitisch aktive Institutionen, Think Tanks und Initiativen, über die eine Einflussnahme von Wirtschaftswissenschaftler*innen auf Ökonomie, Politik und Gesellschaft sowie auf das Denken über deren wechselseitige Beziehungen erfolgt. Eine Aufgabe unserer Untersuchung bestand daher zunächst darin, die Einflussnahme von Ökonom*innen in den letzten Jahrzehnten auf Wirtschaft, Politik und Gesellschaft (über Gremien wie Wirtschaftsministerien, Sachverständigenrat, Wirtschaftsinstitute usw.) sowie das Denken darüber sowohl im universitären Bereich (vermittelt über Lehrbücher, Publikationen, Forschungen) als auch in die Gesellschaft hinein (über

\footnotetext{
1 „Ökonom*innen und Ökonomie“, Projektnr. 2012-575-1, gefördert durch die Hans-Böckler-Stiftung; „Ökonomische Krise und Ökonom*innen“, Projektnr. 14085, gefördert durch die Österreichische Nationalbank.

${ }^{2}$ Wir verwenden eine geschlechterneutrale Schreibweise. Wo wir dennoch von „Ökonomen“ sprechen, handelt es sich tatsächlich nur um männliche Ökonomen.
} 
mediale Präsenz, Kampagnen usw.) zu erfassen. Zentrale Forschungsfrage war, wie und mit welchen Ausrichtungen diese Formung der Ökonomie und des Denkens über ihre wirtschaftspolitische Rolle erfolgte und wer dabei die einflussreichsten Personen und Institutionen waren.

Zur Beantwortung dieser Frage haben wir uns auf die akademischen Vertreter*innen der Ökonomik konzentriert und in einem ersten Schritt eine Datenbank von allen 782 Professor*innen erstellt, die von 1954 bis 1994 in Deutschland in den Bereichen „Volkswirtschaft“ bzw. „Nationalökonomie“, später „Mikro- und Makroökonomie“ sowie „Finanzwissenschaften“ an den Universitäten tätig waren ${ }^{3}$, und zwar mit allen verfügbaren Daten, welche auf performative Einflussmöglichkeiten schließen lassen, wie akademische Herkunft ${ }^{4}$, Lehrer-Schüler-Verhältnisse, Lehrbücher, Publikationen, Gremien, Institutionen, Medienpräsenz ${ }^{5}$ usw. In einem zweiten Schritt wurden diese Daten operationalisiert, zu sechs Wirkungskoeffizienten zusammengefasst ${ }^{6}$ und auf dieser Grundlage der individuelle ,,performative Fußabdruck“ aller Ökonom*innen in unserem Sample berechnet. ${ }^{7}$ Anschließend wurden die Ergebnisse aufbereitet und für eine erste Netzwerkdarstellung ausgewertet.

Die Ergebnisse der Datenanalyse wurden in zwei Formen dargestellt: als Rankings der Ökonom*innen in Tabellen mit den entsprechenden Koeffizienten sowie für die drei wirtschaftspolitisch relevanten Bereiche und ihre wichtigsten Vertreter*innen zusätzlich graphisch in Form von Netzwerkvisualisierungen (ebd., S. $51 \mathrm{ff}$.). Hier wurden die Ökonom*innen mit einem hohen und mittleren medialen Präsenzkoeffizienten, wirtschaftspolitischen Beratungskoeffizienten und wirtschaftspolitischen Akteurskoeffizienten hinsichtlich ihrer Beziehungen zu wirtschaftspolitisch agierenden Institutionen, Think Tanks und Initiativen in bimodalen Netzwerken dargestellt (Knoten sind sowohl Personen als auch Institutionen) (ebd., S. 58, 61, 64). Als wiederkehrendes Muster zeigte sich bei der Visualisierung der hoch gerankten Ökonom*innen ein geteiltes Netzwerk mit zwei Graphen, die dadurch entstanden, dass bei den Ökonom*innen divergierende wirtschaftspolitische Grundeinstellungen bestehen, die sich in entsprechenden institutionellen Verbindungen manifestieren: ein großer Graph mit den Verbindungen von Ökonomen zu marktfundamentalen Insti-

\footnotetext{
$3 \mathrm{Zu}$ einem anderen Herangehen, das die Geschehnisse und das Wirken der daran beteiligten zentralen Akteur*innen und Institutionen in bestimmten einzelnen Zeitabschnitten nachvollzieht, siehe z.B. die Arbeiten von Alexander Nützenadel (2005) und Jan-Otmar Hesse (2010).

4 Wo, wann und bei wem promoviert.

5 Gemessen anhand der Nennungen in „Der Spiegel“ und „Die Zeit“. In einer anderen Studie wurde die mediale Präsenz von Ökonom*innen im Finanzkrisendiskurs von Herbst 2008 bis Sommer 2011 untersucht und die Machtverteilung zwischen diversen Diskurskoalitionen herausgearbeitet (Hirte 2013; Pühringer und Hirte 2015).

6 1. Wissenschaftlicher Produktionskoeffizient, 2. wissenschaftlicher Reproduktionskoeffizient, 3. medialer Präsenzkoeffizient, 4. wirtschaftspolitischer Beratungskoeffizient, 5. wirtschaftspolitischer Akteurskoeffizient und 6. wissenschaftlicher Reputationskoeffizient (Ötsch et al. 2018, S. $19 \mathrm{ff.}$.).

7 Dabei fiel der wissenschaftliche Reputationskoeffizient heraus, da für ihn keine gesicherten und hinreichend einheitlichen Daten verfügbar waren (Ötsch et al. 2018, S. 43). Diese Messung des performativen Fußabdruckes der Ökonom*innen, der dann auch in die Darstellung der Netzwerke eingeht, wird von Sonntag nur beiläufig erwähnt (2019, S. 158).
} 
tutionen, Organisationen und Initiativen und ein kleiner Graph mit einer Verbindung zwischen einem Ökonomen und einem gewerkschaftsnahen Think Tank. ${ }^{8}$

Allerdings waren innerhalb der großen Graphen der Netzwerke zahlreiche Akteure und Institutionen versammelt, welche im Kuhn'schen Verständnis (Kuhn 1976) unterschiedlichen paradigmatischen Schulen der Wirtschaftswissenschaften angehörten. Daher bestand in einem nächsten Schritt die Herausforderung darin, nicht nur formal über institutionelle Zugehörigkeiten, sondern darüber hinaus auch inhaltlich die verbindende Klammer zu bestimmen, d.h. den gemeinsamen „Geist“, in dem die verschiedensten Akteur*innen ihre Zugehörigkeit zu bestimmten Institutionen praktizierten. In Abgrenzung zu den einzelnen paradigmatischen Richtungen innerhalb der Wirtschaftswissenschaften wurde diese gemeinsame ideelle Grundüberzeugung als „Denkstil““ eines ,Denkkollektivs“ bezeichnet (Fleck 1980) und als inhaltliche Klammer das Teilen einer bestimmten Auffassung von „,dem Markt“ eruiert, die diesen zu einem wirkmächtigen realen Akteur überhöht, der wirtschaftlich alles zum Besten richtet (Ötsch et al. 2018, S. 13 ff., 67 ff.). Mit dieser Auffassung geht die radikale Abwertung aller regelnden Institutionen einher, die als marktfremd begriffen werden und daher den sich selbst regulierenden „Markt“ nur stören können, wenn sie ihn nicht gar ,versagen“ lassen. Maximal dürfen diese Institutionen den Markt rahmend begrenzen, wie sich das in der Unterscheidung zwischen Ordnungs- und Prozesspolitik im für die deutsche Wirtschaftspolitik höchst einflussreichen Ordoliberalismus zeigt. Dieses einseitig auf „,den Markt“ fixierte Denken, das den „,freien Markt“ in polarisierender Weise „dem Staat“ und „,der Politik“ gegenüberstellt, wurde deshalb im Buch als „marktfundamental“ bezeichnet. Diesem Denken und den marktfundamentalen Netzwerken gehen wir dann ausführlich in den historischen Kapiteln 4 und 6 unseres Buches nach.

Insgesamt zeigen die Ergebnisse unserer Analyse, dass die Mehrheit jener Ökonomen, die wir mittels der Methode der Messung des performativen Fußabdruckes als potenziell wirkmächtige Ökonomen aus unserem Gesamtsample herausgefiltert haben, in marktfundamentalen Institutionen vernetzt sind. Das sich bei den von uns ausgewählten Koeffizienten wiederholt zeigende Segregationsmuster zweier unverbundener, wenngleich zahlenmäßig sehr ungleichgewichtiger Gruppen bzw. Graphen belegt jedoch auch, dass es stets fortbestehende divergierende Auffassungen und Netzwerke gab.

\section{Zu Sonntags Kritik an der Methodik unserer Netzwerkvisualisierung}

In seiner Kritik an unseren Analyseergebnissen knüpft Nico Sonntag vor allem an diese Visualisierung der zwei unverbundenen Netzwerkgraphen in Abbildung 2 unseres Buches an (Ötsch et al. 2018, S. 58; Sonntag 2019, S. 156ff.). Ihm leuchtet nicht ein, was die genauen Kriterien für die Netzwerkdarstellung waren und warum in ihr Peter Bofinger und seine Verbindung zum gewerkschaftsnahen Wirtschaftsund Sozialwissenschaftlichen Institut (WSI) neben der großen Gruppe an Ökonomen

\footnotetext{
8 Dass in den Tabellen und Graphen nur Ökonomen auftauchen, ist Ausdruck der Tatsache, wie ausgeprägt im Untersuchungszeitraum die Vorherrschaft von Männern in den Diskursen und Netzwerken war.
} 
auftaucht, die in deutschen marktfundamentalen Netzwerken aktiv waren. Er schließt daraus auf eine intransparente, ja willkürliche Auswahl der Ökonomen und ihrer Netzwerkverbindungen in unserer Darstellung. Diesen Vorwurf müssen wir zurückweisen. Der Auswahl der Ökonom*innen und der Darstellung ihrer Netzwerkverbindungen lagen folgende zwei Kriterien zugrunde: Es wurden nur Ökonom*innen berücksichtigt, die hinsichtlich ihres performativen Fußabdruckes mindestens einen hohen oder mittleren medialen Präsenzkoeffizienten aufwiesen und in wirtschaftspolitisch agierenden Institutionen, Think Tanks und Initiativen aktiv waren (Ötsch et al. 2018, S. 57). Mit Letzteren bilden wir die einflussrelevanten institutionellen Verbindungen der Ökonom*innen ab. Unter diesen Verbindungen werden Funktionen und Mitgliedschaften in den entsprechenden Institutionen und Organisationen sowie die aktive Unterstützung von Initiativen und Appellen verstanden (ebd., u.a. S. 224 f., Fußnote 263). Die Kanten zeigen solche Verbindungen zwischen Personen und bestimmten Netzwerken. Deshalb taucht Bofinger zwangsläufig in der Abbildung 2 auf. Das dient nicht ,nur illustrativen Zwecken“, wie Sonntag vermutet (2019, S. 158). Und deshalb fehlen von den 22 Ökonom*innen, die wie Bofinger einen entsprechenden, hinreichend hohen Koeffizienten aufweisen, all jene in der Abbildung, die nicht über solche Verbindungen zu Institutionen, Think Tanks und Initiativen verfügen, egal welcher wirtschaftspolitischen Ausrichtung diese sind. ${ }^{9}$ Es war also nicht die Absicht, nur selektiv die Verbindungen von Ökonom*innen zu marktfundamentalen Institutionen bzw. Netzwerken darzustellen und Bofinger bloß als Kontrastfall ,einzuspielen“. Die betreffende Abbildung gibt einfach die faktische institutionelle Vernetzung unseres Subsamples wieder. Das auffällige zahlenmäßige Missverhältnis zwischen jenen einflussreichen Ökonomen, die sich in marktfundamentalen Netzwerken engagieren, und jenen, die sich in anderen, in diesem Fall gewerkschaftsnahen Netzwerken engagieren, ist kein Artefakt, sondern ergibt sich zwangsläufig aus den von uns für diese Netzwerkabbildung herangezogenen Daten und zwei Kriterien.

Deshalb haben wir auch keine Zentralitätsmaße oder Cluster berechnet; unsere Analyse ist keine quantitativ-formalanalytische, sondern eine datengestützt beschreibende. ${ }^{10}$ Die Netzwerkabbildungen in Kapitel 2 unseres Buches bilden nur einen Baustein bei der Rekonstruktion der Vernetzungsprozesse der Ökonom*innen unseres Samples, wenn auch keinen unwichtigen. Deshalb fanden nicht nur Organisationen und formale Mitgliedschaften Eingang in unsere Untersuchung, sondern auch Funktionen und die Unterstützung bestimmter Initiativen, wie des Hamburger Appells, weil es uns darum ging, die Einflussnahme von Ökonom*innen auf wirtschaftspolitisch relevante Bereiche möglichst breit (wenn auch nicht vollständig)

\footnotetext{
9 Nach analogen Kriterien, wenngleich auf Basis anderer ausgewählter Koeffizienten des performativen Fußabdruckes, wurden auch die Netzwerkabbildungen 4, 6 und 23 in unserem Buch erstellt. Bei der von Sonntag kritisierten Netzwerkkante zwischen einer Institution und einer Initiative in Abbildung 2 handelt es sich um einen bedauerlichen technischen Darstellungsfehler. Die Verbindungslinie zwischen Carl-Christian von Weizsäcker und dem Hamburger Appell geht fälschlicherweise durch die Ludwig-Erhard-Stiftung hindurch und stiftet auf diese Weise optisch zwei falsche Verbindungen. Für eine richtige Darstellung siehe Abbildung 23 (Ötsch et al. 2018, S. 251). Wir bitten, diesen Fehler zu entschuldigen.

$10 \mathrm{Zu}$ einer jüngeren Netzwerkanalyse, in der auch Zentralitätsmaße usw. berechnet wurden, siehe Pühringer und Beyer 2021.
} 
zu erfassen. Gerade Initiativen und Appelle folgen einer ,logic of connective action", bei der statt formalen Bindungen das selbstmotivierte Teilen und Mitteilen von bereits verinnerlichten Ideen im Vordergrund steht (Benett und Segerberg 2012). Da es uns zudem vornehmlich um die Geschichte ordoliberaler Netzwerkbildungen in Deutschland ging, stand für uns in Kapitel 2 auch nicht die Abbildung eines Echtzeitnetzwerkes zu einem bestimmten Zeitpunkt im Vordergrund, sondern die Rekonstruktion der kumulativen bzw. sukzessiven Vernetzung der einflussreichsten Ökonom*innen über den ganzen Untersuchungszeitraum bis in die jüngere Gegenwart hinein. Deshalb enthalten unsere Netzwerkgraphen ggf. auch Personen, die sich nicht persönlich kannten bzw. aufgrund des großen Altersunterschiedes auch nicht persönlich kennen konnten. Die Persistenz des ökonomischen und wirtschaftspolitischen Denkens wird, wie der Denkstil in anderen Bereichen der Gesellschaft auch, maßgeblich durch das Wirken von Organisationen und Institutionen und den institutionellen Verbindungen der Akteure zu ihnen gesichert, also nicht allein durch direkte soziale Interaktionen zwischen Personen.

\section{Schluss: Über die Gefährlichkeit von Netzwerkabbildungen und die Verantwortung von Wissenschaftler*innen}

Sonntag warnt vor den Gefahren der Reifizierung von Knoten und Kanten zu unmittelbaren Abbildern sozialer Tatbestände (Sonntag 2019, S. 153f.) sowie dem angeblich großen Verführungspotenzial der Netzwerkanalyse und von Netzwerkabbildungen (ebd., S. 167 f.). Dies tut er in einer insinuierenden Weise, die nahelegt, unsere ,unsaubere“ Netzwerkanalyse hätte sich dieser Vergehen wenn schon nicht direkt schuldig, so doch indirekt mitschuldig gemacht, und er erinnert in diesem Zusammenhang an die „Zusätzliche Verantwortlichkeit“ der Wissenschaftler*innen, „weil ihre Forschung von der Öffentlichkeit rezipiert wird“ (ebd., S. 167). Mit diesem Hinweis auf die gesellschaftspolitische Sensibilität gerade solcher Forschungen wie der unsrigen rennt der Autor jedoch bei uns nur offene Türen ein. Dieser Verantwortlichkeit gegenüber der Öffentlichkeit sind wir uns durchaus bewusst. Äußerst merkwürdig mutet daher der Unterton von Sonntags Mahnung an, man solle die Qualität von wissenschaftlichen Untersuchungen auch danach bewerten, ob sie in der Öffentlichkeit unliebsame Folgen zeitigen, falsch verstanden oder missbräuchlich verwendet werden (können). Wer sich eines solchen diffusen Argumentes über die "Gefahr“ möglicher ,falsche[r] Schlussfolgerungen über den Einfluss und die Stellung von Akteuren" bedient (ebd.), müsste konsequenterweise vor der Gefährlichkeit der Sozialwissenschaften überhaupt warnen. Denn auch die seriösesten Forschungsergebnisse können aus Sicht bestimmter Interessengruppen gesellschaftspolitisch „gefährlich“ sein und deren Forderung begründen helfen, sie besser nicht öffentlich zu machen.

Ein jüngeres Beispiel hierfür ist die Debatte um die Studie von Uwe Krüger zu Journalisten-Netzwerken und deren Einflüsse auf inhaltliche Äußerungen zur deutschen Außenpolitik in deutschen Leitmedien (Krüger 2019), deren Ergebnisse von den ZDF-Kabarettisten der „Anstalt“ herangezogen wurden, um die Verbindungen führender deutscher Journalist*innen mit US-nahen Denkfabriken einem breiten Pu- 
blikum zugänglich zu machen. Die Klagen gegen diese Veröffentlichung scheiterten zwar, und ebenso wurde der Argumentation der „Unwissenschaftlichkeit“ der Arbeit begegnet (Schreyer 2021). Aber im Kern sind diese Tendenzen zur Unterdrückung unliebsamer kritischer Untersuchungen eine Bestätigung des Anspruchs, der mit der Netzwerkforschung einhergeht: mit der Analyse sozialer Netzwerke über die soziale Realität aufzuklären. Die Einlösung dieses Anspruches wird jedoch gefährdet, wenn wissenschaftliche Netzwerkanalysen nicht als Strukturanalysen begriffen werden, sondern nur als eine mehr oder weniger geglückte Aufdeckung kollektiver Strategien von Allianzbildung. ${ }^{11}$

Die Wissenschaftler*innen innerhalb der Scientific Community - und also auch wir - wissen um den grundsätzlichen Modellcharakter sowie die Methoden- und Kontextgebundenheit allen wissenschaftlichen Wissens. Das kann aber nicht heißen, die Fachkommunikation solle unter dem Vorbehalt stehen, dass in ihr nur noch gesagt und abgebildet werden darf, was in der Öffentlichkeit, die ganz anderen kommunikativen Regeln und Aufmerksamkeiten folgt, nicht missverstanden oder für unlautere Zwecke gebraucht werden kann. Das wäre das Ende der Wissenschaft. Sonntag hat uns in seiner Kritik vorgeworfen, wir hätten solche wissenschaftlich nicht gedeckten „lediglich insinuierten Schlussfolgerungen“ in unserer Untersuchung gezogen (Sonntag 2019, S. 162). So etwas zu behaupten und gleichzeitig selbst zu insinuieren, visuelle Netzwerkabbildungen - inklusive der unsrigen? -, könnten ,schnell zu einem verschwörungstheoretischen Denken anregen, dass es obskure Netzwerke seien, die allein über die Ausbreitung und Hegemonie von Ideen und die politische Macht im Land entscheiden" (ebd., S. 168), ist - zumal in einem Fachjournalartikel - kein guter Stil. Daher müssen wir diesen Vorwurf an den Autor der Kritik zurückverweisen.

Funding Open Access funding enabled and organized by Projekt DEAL. Stephan Pühringer bedankt sich für finanzielle Unterstützung beim FWF (Projektnummer ZK60-G27).

Open Access Dieser Artikel wird unter der Creative Commons Namensnennung 4.0 International Lizenz veröffentlicht, welche die Nutzung, Vervielfältigung, Bearbeitung, Verbreitung und Wiedergabe in jeglichem Medium und Format erlaubt, sofern Sie den/die ursprünglichen Autor(en) und die Quelle ordnungsgemäß nennen, einen Link zur Creative Commons Lizenz beifügen und angeben, ob Änderungen vorgenommen wurden.

Die in diesem Artikel enthaltenen Bilder und sonstiges Drittmaterial unterliegen ebenfalls der genannten Creative Commons Lizenz, sofern sich aus der Abbildungslegende nichts anderes ergibt. Sofern das betreffende Material nicht unter der genannten Creative Commons Lizenz steht und die betreffende Handlung nicht nach gesetzlichen Vorschriften erlaubt ist, ist für die oben aufgeführten Weiterverwendungen des Materials die Einwilligung des jeweiligen Rechteinhabers einzuholen.

Weitere Details zur Lizenz entnehmen Sie bitte der Lizenzinformation auf http://creativecommons.org/ licenses/by/4.0/deed.de.

11 Vor einer „Überinterpretation von Netzwerkmustern“ als Ausdruck kollektiver Strategien der Allianzbildung, ohne ,einen Beleg für ihren bewussten oder konzertierten Charakter“ beizubringen, warnt auch Claire Lemercier (2012, S. 20). 


\section{Literatur}

Benett, W. L., \& Segerberg, A. (2012). The logic of connective action. Information, Communication \& Society, 15, 739-768.

Emirbayer, M. (1997). Manifesto for a relational sociology. American Journal of Sociology, 103, $281-317$.

Fleck, L. (1980). Entstehung und Entwicklung einer wissenschaftlichen Tatsache. Einführung in die Lehre vom Denkstil und Denkkollektiv. Frankfurt a. M.: Suhrkamp.

Haas, J., \& Malang, T. (2010). Beziehungen und Kanten. In C. Stegbauer \& R. Häußling (Hrsg.), Handbuch Netzwerkforschung (S. 89-98). Wiesbaden: VS Verlag für Sozialwissenschaften.

Hesse, J.-O. (2010). Wirtschaft als Wissenschaft. Die Volkswirtschaftslehre in der frühen Bundesrepublik. Frankfurt a. M.: Campus.

Hirte, K. (2013). ÖkonomInnen in der Finanzkrise. Diskurse, Netzwerke, Initiativen. Marburg: Metropolis. Krüger, U. (2019). Meinungsmacht. Der Einfluss von Eliten auf Leitmedien und Alpha-Journalisten - eine kritische Netzwerkanalyse. 2., erw. und überarb. Aufl. Köln: Herbert von Halem.

Kuhn, T. S. (1976). Die Struktur wissenschaftlicher Revolutionen. Frankfurt a. M.: Suhrkamp.

Lemercier, C. (2012). Formale Methoden der Netzwerkanalyse in den Geschichtswissenschaften: Warum und Wie? Österreichische Zeitschrift für Geschichte, 23, 16-41.

Nützenadel, A. (2005). Stunde der Ökonomen. Wissenschaft, Politik und Expertenkultur in der Bundesrepublik 1949-1974. Göttingen: Vandenhoeck \& Ruprecht.

Ötsch, W. O., Pühringer, S., \& Hirte, K. (2018). Netzwerke des Marktes. Ordoliberalismus als Politische Ökonomie. Wiesbaden: Springer VS.

Pühringer, S., \& Beyer, K. (2021). Who are these economists Germany might listen to? In J. Maesse, S. Pühringer, T. Rossier \& P. Benz (Hrsg.), Power and influence of economists: Contributions to the social studies of economics (S. 147-169). London: Routledge.

Pühringer, S., \& Hirte, K. (2015). The financial crisis as a heart attack. Discourse profiles of economists in the financial crisis. Journal of Language and Politics, 14, 599-625.

Saunders, C. (2007). Using social network analysis to explore social movements: A relational approach. Social Movement Studies, 6, 227-243.

Schreyer, P. (2021). Zwischen Lesern und Lobbynetzwerken. Telepolis, 09. Juli 2021. https://www. heise.de/tp/features/Zwischen-Lesern-und-Lobbynetzwerken-3368298.html?view=print. Zugegriffen: Dez. 2021.

Sonntag, N. (2019). Anmerkungen zum Gebrauch der Netzwerkanalyse in der Neoliberalismusforschung. Berliner Journal für Soziologie, 29, 151-169.

Stegbauer, C. (2010). Positionen und positionale Systeme. In C. Stegbauer \& R. Häußling (Hrsg.), Handbuch Netzwerkforschung (S. 135-144). Wiesbaden: VS Verlag für Sozialwissenschaften.

Walter Otto Ötsch geb. 1950. Professor für Ökonomie und Kulturgeschichte an der Cusanus Hochschule für Gesellschaftsgestaltung Koblenz. Forschungsschwerpunkte: Kultur- und Wirkungsgeschichte ökonomischer Theorien, Rechtspopulismus und Neoliberalismus, Aspekte einer imaginativen Politischen Ökonomie. Aktuelle Veröffentlichungen: (mit N. Horaczek) Wir wollen unsere Zukunft zurück! Eine Streitschrift für mehr Phantasie in der Politik, 2021; (mit T. Steffestun, Hrsg.) Wissen und Nichtwissen der ökonomisierten Gesellschaft. Aufgaben einer neuen Politischen Ökonomie, 2021; (mit S. Graupe, Hrsg.) Imagination und Bildlichkeit der Wirtschaft. Zur Geschichte und Aktualität imaginativer Fähigkeiten in der Ökonomie, 2020.

Katrin Hirte geb. 1964. PD Dr., wissenschaftliche Mitarbeiterin am Institut für die Gesamtanalyse der Wirtschaft, Johannes Kepler Universität Linz. Forschungsschwerpunkte: Politische Ökonomie, Ambivalenz zwischen Wirtschaftsentwicklung und Entwicklung des ökonomischen Denkens, Diskurs- und Netzwerkanalyse, Transformationsforschung. Aktuelle Veröffentlichungen: Unternehmenskonzentrationen in der Fleischbranche und die performative Rolle der Agrarökonomik - das Beispiel Sektorplanungen im Schlachthofbereich, in: Österreichische Zeitschrift für Soziologie, 2021; Das doppelte Reflexionsproblem, in: L. Hochmann (Hrsg.), Economists4future. Verantwortung übernehmen für eine bessere Welt, 2020; Friedmans Instrumentalismus und das Problem von Kopernikus. Zur zentralen Rolle von Ausgangsannahmen in Theorien, in: S. Pühringer, S. Graupe, K. Hirte, J. Kapeller, S. Panther (Hrsg.), Jenseits der Konventionen: Alternatives Denken zu Wirtschaft, Gesellschaft und Politik, 2020. 
Stephan Pühringer geb. 1985. Dr., wissenschaftlicher Mitarbeiter am Institut für die Gesamtanalyse der Wirtschaft, Johannes Kepler Universität Linz. Forschungsschwerpunkte: Politische Ökonomie, Social Studies of Economics, Neoliberalismusforschung, Wirkungsgeschichte ökonomischen Denkens. Aktuelle Veröffentlichungen: Zur Pluralität der ökonomischen Politikberatung in Deutschland. Eine empirische Untersuchung, in: S. Büttner und T. Laux (Hrsg.), Umstrittene Expertise. Zur Wissensproblematik der Politik. Leviathan, Sonderband 38, 2021; (mit K. Beyer) Who are these economists Germany might listen to?, in: J. Maesse, S. Pühringer, T. Rossier und P. Benz (Hrsg.), Power and influence of economists: Contributions to the social studies of economics, 2021; (mit J. Kapeller und C. Grimm) Paradigms and policies: The current state of economics in the German-speaking countries, in: Review of International Political Economy, 2021. 